\title{
Teaching Reform and Research of Medical Physics Course under the Background of Massive Open Online Course
}

\author{
Wenbin Kang ${ }^{1,3,{ }^{*}, \text { Baiping } \text { Liu }^{2} \text {,Pengcheng Zhang }}{ }^{1,3}$,Benchao Zhu1 ${ }^{\text {,3 }}$, \\ Guangxiao $\mathrm{He}^{1,3}$ and Jia Guo ${ }^{1,3}$ \\ ${ }^{1}$ School of Public Health and Management, Hubei University of Medicine, Hubei Shiyan 442000, China; \\ ${ }^{2}$ Dongfeng Senior High School, Shiyan 442008, China; \\ ${ }^{3}$ Bio-X research center, Hubei University of Medicine, Hubei Shiyan 442000, China. \\ wbkang@hbmu.edu.cn \\ *The corresponding author
}

Keyword: Outstanding physician education; Medical physics; MOOC; Teaching reform

Abstract. A series of teaching reform and teaching practice of medical physics course are proposed based on the background of massive open online course (MOOC) and excellent doctor education, which combined with the characteristics of medical physics course. The revelation of MOOCs, in coming for medical physics teaching reformation is to change teaching concept and learning methods, construct new teaching and learning mode, construct network-sharing platform of medical physics course and explore flipped classroom teaching reformation of medical physics. The reform and practice of teaching methods and teaching ways are shown, which obtained some good teaching effect. It is a beneficial exploration for the positive and reasonable use of network resources and construction of a new education system of medical physics teaching.

\section{卓越医生教育背景下《医学物理学》慕课体系构建 与研究}

\author{
康文斌 $^{1,3}$, 刘百萍 ${ }^{2}$, 张鹏程 ${ }^{1,3}$, 朱本超 ${ }^{1,3}$, 何光晓 ${ }^{1,3}$, 郭佳 ${ }^{1,3}$ \\ ${ }^{1}$ 湖北医药学院，公共卫生与管理学院，湖北十堰 442000 \\ 2 东风高级中学, 湖北十堰 442001 \\ 3 湖北医药学院, Bio-X 研究中心, 湖北十堰 442000
}

摘要: 本文基于卓越医生教育背景下, 结合医学物理学课程内容特点和教学实践, 对医学物理学课程的教学模式、教学 方式和教学方法的改革进行了研究与实践。慕课来袭对《医学物理学》教学改革的启示是改变教学理念和学习方法，构建新 型的教学模式, 建设医学物理学网络共享平台课程, 探索医学物理学的翻转课堂教学改革。取得了良好的教学效果, 为医学 物理学教学积极合理使用网络资源与新型教育体系的构建进行了有益的探索。

关键词: 卓越医生教育; 医学物理学; 慕课; 教学改革

中图分类号: G 642.0 文献标志码: A

\section{1 引言}

医学物理学是一门将物理学原理、理论、方法和技术等应用于医学诊断与治疗的学科, 是医药类院校 本专科生必修的基础理论课之一 错误! 未找到引用源。。医学物理学的教学对培养学生综合能力有着其他 课程无法替代的作用。因此，要根据卓越医生培养计划的形式和实际情况，不断研究和探索新方法，提高 
医学物理学教学质量, 培养高素质的医学人才。

随着教育改革的深入进行，教育部、卫生部适时提出了在全国医药学各专业实施 “卓越医生”教育的 理念 0 。此外, 大规模在线开放课程（Massive Open Online Courses, M00Cs) 自 2013 年以来日益受到中 国大学高等教育界的瞩目 [5-10]。互联网时代, MOOCs 给传统教育和大学功能带来了巨大的影响, 推动了 教学模式的改革与创新, 为提升教育质量提供了新思路、新方法和新机遇。如何紧跟世界潮流, 让慕课成 为高等教育的一个重要部分, 建设具有医药特色的医学物理学慕课课程是我们医学物理学教师义不容辞的 责任, 并以此提供给医药学类专业在校生课堂内外的学习平台, 正是我们教学的改革目标之一。目前, 湖 北省尚未有高校在卓越医生教育和慕课背景下, 对医学物理学课程慕课化进行教学改革与研究实践 [10-16]。作为一门重要的基础必修课, 如何在卓越医生教育和慕课背景下进行《医学物理学》课程的改 革与建设, 全面提升医学生的科学素养和创新能力, 这需要我们对《医学物理学》教学进行比较深入的研 究和实践, 以适应教育改革的时代要求。

\section{2 医学物理学教学现状及存在的问题}

\section{1 理论学时少、教学内容分散, 扩展知识点不易展开}

医学物理学的内容覆盖了物理学中力学、热学、电磁学、流体力学、振动、波动、光学和近代物理在 医学中的应用等内容, 教学内容较为分散, 章与章之间的关联性较低。这些教学内容在理工科院校的课时 一般在 108 学时以上。据调查统计, 在全国近 105 所医药院校内, $72 \%$ 被调查院校的医学物理学理论课学 时数不足 60 学时, 而我校的医学物理学理论教学学时数仅为 32 学时。如何在较少课时内完成繁重的教学 内容成为医学物理学课程教学的难题。

\section{2 学生学习积极性不高, 课前预习及课后复习不够}

医学物理学课程在医药院校中一般为必修考查类课程, 知识点与医学主干课较远, 学生通常不能对此 课程引起足够的重视, 缺乏自主学习的兴趣, 学生对医学物理学课程课前不预习, 课后很少复习。对课程 的理解慢且掌握不够。同时, 在传统的教学模式中, 教师主要以讲授法为主, 学生被动听课, 教师与学生 缺少有效的互动。很多学生对医学物理学的学习只是停留在课堂上教师的授课内容。学生很难将医学物理 学应用于其他相关学科的学习之中。在传统的教学过程中, 教学的时间通常是比较固定的。但各学生的作 息时间不同，学生很难自主选择自己效率较高的时间来学习课程。

\section{3 实验课时紧张, 实验条件有限}

据调查, 在全国近 105 所医药院校内 $70 \%$ 被调查院校的医学物理学实验课学时数低于 30 学时, 我校 医学物理学实验课时仅为 18 学时。同时, 医学物理学实验的经费投入有限, 实验设备相对陈旧, 严重地 制约了实验内容的更新。

\section{3 医学物理学慕课课程建设}

\section{1 医学物理学 MOOCs 教学内容和教学方式的重构}

与传统的教学模式不同, MOOC 教学模式可以提供在线学习资源。在医学物理学中的 MOOC 教学实践中, 我们采用线上与线下教学相结合的教学方式。对教学内容和教学方式进行重构。首先, 我们对教学内容进 行了划分, 将预习、知识点的讲解、讨论、课后作业和测验等环节移到线上进行。在线下的课堂教学中, 教师主要对重点和难点的内容进行总结与讲解, 并对学生提出的问题进行讨论与答疑。在 MOOC 教学模式 
中, 需要将每一知识点做成独立的教学视频。才能方便学生进行学习。在制作教学视频方面, 我们首先在 原有的多媒体课件的基础上, 将每一个知识点做成独立的课件。之后我们采用录屏的方式将课件制作成视 频, 并用摄像头录制教师讲解的过程。最后运用视频剪辑软件将课件与教师讲解过程合并生成各个知识点 独立的教学视频。

\section{2 医学物理学 MOOC 教学设计}

(1) 合理安排教学内容。提高学生独立学习能力在每次上课前, 我们将可以激发学生思考的预习资 料及每个知识点的课程视频及教学课件发布于课程平台上, 供学生自主学习与理解。同时, 我们还对一些 课上不做重点讲解的知识点加上扩展链接, 提供更丰富的网络资源, 供有余力的学生开拓视野。

（2）增添计算模拟仿真实验, 提高学生分析解决问题能力。在课程平台上增加了计算模拟实验来增 强学生对知识点的理解。同时, 学生可以模拟实验环境上操作由于经费限制不能真实操作的实验, 在一定 程度上缓解经费限制对实验教学的影响。

(3) 强课程反馈, 改进教学方法与策略我们在课程平台上设置有论坛, 学生在自主学习的过程中, 遇 到不明白的知识点可以在论坛中及时反馈。

\section{3 医学物理学慕课化的优势}

(1) MOOCs 将引领教师在《医学物理学》教学理念和方法上产生巨变。近年来《医学物理学》教学方 面一直都在尝试改革, 但最终并没有改变教师 “教” 的主导地位, 学生的主体地位张力不够, 以 “学” 为 本的教学价值取向并未落到实处。MOOCs 突出了学生自主学习的地位, 而教师不得不面对的问题是如果学 生可以在网上随时随地看到名家的《医学物理学》课程, 那么教师在课堂上该如何讲解才能吸引学生的兴 趣? 这将迫使教师不得不变革教学理念和创新教学方法, 从而进一步提高自身水平和教学质量, 最终使学 生从中受益。

MOOCs 使教学的本质充分体现出来, 可有效解决教与学之间的矛盾。网络课程是当代年轻人喜欢的方 式, 扩大了学生学习的空间, 提高了自主学习的能力。采用混合式教学, 翻转课堂, 依赖网络组织学习, 配合答疑课、讨论课, 鼓励学生走上讲台, 促进学生自我探究式学习、小组协作式学习, 从而提升学生思 考辨析力、学习能力、表达能力和协作能力 0 。

(2) MOOCs 改变学生学习《医学物理学》的方法。MOOCs 时代, 学生可以在网上搜寻相关的《医学物 理学》教学视频, 从中学习自己最需要、最感兴趣的部分, 激发他们学习《医学物理学》的积极性和主动 性。更为重要的是, 他们可以跳过已经掌握的部分, 重复播放难懂的部分, 并且启迪思考, 与名家互动。 学生从被动变为主动, 从 “要我学” 变成 “我要学”, 大大提高了学习效果。

(3) MOOCs 能够促进医学物理教学团队的建设, 实现优质教育资源共建共享、协同发展当前医学物理 学面临的问题不是哪个学校短时间内可以自我完善的, 最有效的解决对策就是高质量课程资源的共享。信 息时代先进的网络技术为资源共享提供了理想的途径，使优质教育资源的共享成为时代的必然。

\section{4 总结}

《医学物理学》未来的教学改革方向应该以 MOOCs 为契机, 借助 MOOCs 资源共享平台, 使课内教学和 课外自主学习相得益彰, 从根本上提高教学质量并让学生从中受益。利用 MOOC 教学模式对医学物理学教 学进行改进, 通过合理安排教学内容, 提供多类型的学习资源, 增添计算模拟仿真实验和加强课程反馈等 
方式, 提高学生学习积极性, 培养学生自主学习能力、分析解决问题能力。我们相信, 医学物理学慕课课 程在培养医学学生的自主学习和协作学习中具有非常重要的作用, 对学生综合能力的培养也将发挥很大的 作用。

\section{5 致谢}

本研究得到 2017 年国家级大学生创新创业训练计划项目（批准号：201710929002)。

\section{Acknowledgement:}

We thank the supports from the2017 National Training Program of Innovation and Entrepreneurship for Undergraduates (Grant Nos. 201710929002).

\section{References}

[1] Medical Physics [J]. Yale Journal of Biology \& Medicine, 1950, 23.

[2] Weiyuan C. China's village doctors take great strides [J]. Bulletin of the World Health Organization, 2008, 86(12):914.

[3] Elosegi A, Gessner M O, Young R G. River doctors: Learning from medicine to improve ecosystem management [J]. Science of the Total Environment, 2017, 595:294.

[4] Tang W Y, Yun-Tao L I, Diao Z Q, et al. The Reform and Construction of Medical Physics Course under the Education Background of Excellent Doctors [J]. Chinese Journal of Medical Physics, 2012, 29(6):3837-3840.

[5] Ira G, Brian K, Yager J D, et al. Massive Open Online Courses in Public Health[J]. Frontiers in Public Health, 2013, 1:59.

[6] Goldberg L R, Crocombe L A. Advances in medical education and practice: role of massive open online courses [J]. Advances in Medical Education \& Practice, 2017, 8:603-609.

[7] Rodriguez C O. MOOCs and the AI-Stanford Like Courses: Two Successful and Distinct Course Formats for Massive Open Online Courses [J]. European Journal of Open, Distance and E-Learning, 2012:13.

[8] Margaryan A, Bianco M, Littlejohn A. Instructional quality of Massive Open Online Courses (MOOCs)[J]. Computers \& Education, 2015, 80:77-83.

[9] Hew K F, Cheung W S. Students' and instructors' use of massive open online courses (MOOCs): Motivations and challenges [J]. Educational Research Review, 2014, 12:45-58.

[10] Rodriguez O. The Concept of Openness behind c- and x-MOOCs (Massive Open Online Courses) [J]. Open Praxis, 2013, 5(1): 67-73.

[11] Kellogg S. Online learning: How to make a MOOC [J]. Nature, 2013, 499(7458):369-371.

[12] Mcauley A, Stewart B, Cormier D, et al. The MOOC Model for Digital Practice [J]. 2010.

[13] MOOC scholarship [J]. Learning Media \& Technology, 2014, 39(3):328-345.

[14] Breslow L, Pritchard D E, Deboer J, et al. Studying Learning in the Worldwide Classroom Research into edX's First MOOC[J]. Research \& Practice in Assessment, 2013, 8:13-25.

[15] King C, Robinson A, Vickers J. Online education: Targeted MOOC captivates students [J]. Nature, 2014, 505(7481):26.

[16] Ebben M, Murphy J S. Unpacking MOOC scholarly discourse: a review of nascent.

[17] Kononowicz A A, Berman A H, Natalia S, et al. Virtual Patients in a Behavioral Medicine Massive Open Online Course (MOOC): A Case-Based Analysis of Technical Capacity and User Navigation Pathways:[J]. Jmir Medical Education, 2015, 1(2):e8. 